\title{
The numerical simulation of enhanced heat transfer on a Linear Fresnel molten salt-type receiver tube filled with porous media
}

\author{
Chenggang Yang ${ }^{1}$, Yuning Zhang ${ }^{2}$, Fenghe Yan ${ }^{1 *}$,Wenguang Zhang ${ }^{1}$, Wei $\mathrm{Li}^{2}$ \\ ${ }^{1}$ Nuclear and Radiation Safety Centre MEE, Beijing 102400, P.R. China \\ ${ }^{2}$ Beijing TeraSolar Photothermal Technologies Co., Ltd, Beijing 101102, P.R. China
}

\begin{abstract}
In this paper, three-dimensional numerical simulation was taken on a Linear Fresnel solar receiver tube using molten salt as heat transfer fluid (HTF), in which the porous media was filled to enhance the heat transfer efficiency. The simulation was to analyze the influence of the different conditions (filling rate, porosity and thermal conductivity) on heat transfer effect and wall temperature difference. The results revealed that the $\mathrm{Nu}$ (Nusselt number) increased firstly and then decreased with the increasing filling rate in both center filling and annular filling types. The optimal thermal performance were obtained when filling rate were 0.8 and 0.2 in center filling and annular filling, respectively. The $\mathrm{Nu}$ were about 1.7 and 1.5 times as the clear receiver. The circumferential temperature difference decreased firstly and then increased with filling rate increasing in both center filling and annular filling types. The lowest circumferential temperature differences were achieved at the filling rate 0.8 and 0.4 in center filling and annular filling types, and temperature difference decreased $15.88^{\circ} \mathrm{C}$ and $22^{\circ} \mathrm{C}$ compared with clear receiver, respectively. The Nu and PEC both decreased with porosity increasing. However, the thermal conductivity of porous media had little influence to the $\mathrm{Nu}$ and circumferential wall temperature.
\end{abstract}

\section{Introduction}

The solar energy thermal power generation is attracting more and more attention due to its advantage of low cost and clean characteristics, under the background of increasingly serious global environmental pollution and energy issues ${ }^{[1]}$. Solar receiver tube is the core component in the process of energy transformation. The non-uniform heat flux distribution can cause the large temperature field in the receiver tube, even damage of component and decrease of working life. So far, the trough-based and power-tower are the most used systems in research and application of solar power plants. Compared with trough-based receiver, traditional Linear Fresnel Reflector, with no rotating joint in high temperature parts, is of low cost and high safety property [2]. Because the receiver tube is non-uniformed distributed on the surface, the flow heat transfer characteristics is to be studied in order to ensure the safety of practical operation.

Lots of previous studies have been done on the aspect of heat transfer enhancement of collector tubes. Chang et al. ${ }^{[3]}$ investigated the enhanced heat transfer by using molten salt in the tube under the circumferential nonuniform heat flux boundary conditions. Wang et al. ${ }^{[4]}$ analyzed the resistance characteristics and heat transfer effect of built-in ligament in the corrugated pipe. Yan et al. ${ }^{5]}$ adopted the numerical simulation to analyze the DSG heat collector tube and found that internally ribbed tube could markedly enhance the heat transfer property and decrease the temperature of tube wall. Porous media is regarded as a new type enhanced heat transfer material, due to its high specific surface area and high thermal conductivity. Wang et al. ${ }^{[6,7]}$ studied the porous media in a trough collector tube with water or steam as working medium. The results revealed that the porous media could significantly reduce the mean temperature difference between light concentration part and nonconcentration part, and the temperature difference of circumferential of tube wall was decreasing with the increase of filling ratio of porous media. Kumar et al. ${ }^{[8]}$ found that the heat transfer performance was improved by $64.3 \%$ by fixing porous media into the trough heat collector. The optimized filling method of porous media in the collector could be calculated by coupled genetic algorithm and CFD simulation by ZHENG ${ }^{[9]}$. However, the porous media will inevitably increase the flow resistance. To further enhance the heat transfer property, more systematic researches are to be carried out by optimizing the parameters of porous media.

Currently, there are few researches on the filling of porous media in Fresnel collector tubes with molten salt as heat transfer medium. In this paper, three-dimensional

*Corresponding author: 532182933@qq.com 
numerical simulation method was used to study the influence of two typical filling methods of porous medium (including filling ratio and operation parameters) in the tube to the flow heat transfer and wall temperature, with molten salt as heat transfer medium. The purpose of this study is to provide guidance to the application of porous media in Fresnel-type molten salt solar power station.

\section{Introduction of the model}

In linear Fresnel light concentration system, sunlight will be repeatedly reflected by strip-shaped plane mirrors and enter the top of the heat receiver, then reflected by CPC and reach the surface of the tubes by penetrating the glass tube. The sunlight will be absorbed by surface coating and transformed into heat energy. Then the heat can be transmitted to the inner wall through the tube wall of the heat absorbing tube, and the working medium in the tube will be heated. As shown in Fig. 1, the receiver tube is filled with a porous medium (copper foam, aluminum foam, etc.) in two methods, in-filling porous insert and out-filling porous insert. The parameters of receiver tube were set as: Length $\mathrm{L}=2000 \mathrm{~mm}$, inside diameter $D_{\mathrm{i}}=26 \mathrm{~mm}$, wall thickness $\delta=2 \mathrm{~mm}$, thermal conductivity coefficient $\lambda=18.4 \mathrm{~W} / \mathrm{m} / \mathrm{K}$. The following assumptions were used to simply the calculations: (1) Steady flow; (2) Neglecting the natural convection and radiation heat transfer in the tube and heat loss outside the tube; (3) The porous media is uniform and isotropic; (4) The porous framework and fluid satisfy local heat balance; (5) Neglecting thermal contact resistance between receiver tube and porous media in out-filling situation.

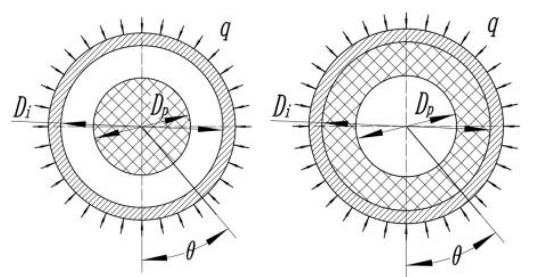

Fig.1.Cross-section of receiver tube filled with porous medium (a) In-filling porous insert and filling ratio: $Y_{c}=\frac{D_{p}}{D_{i}} b$,

(b) Out-filling porous insert and filling ratio: $Y_{o}=\frac{D_{i}-D_{p}}{D_{i}}$.

\section{Calculation method and verification}

\subsection{Calculation method}

The calculation domain of numerical simulation includes receiver tube solid domain, inner fluid domain and porous domain. The numerical calculation used Reynolds time-averaged N-S governing equation to discretize the governing equation based on the finite volume method. The turbulence model adopted the standard k-e model, and steady-state governing equations for flow and heat transfer is defined as equation (1):

$$
\nabla \cdot(\rho u \Phi)=\nabla \cdot(\Gamma \nabla \Phi)+S
$$

Where, $\Gamma$-Generalized diffusion coefficient, $\mathrm{S}-$ Generalized source term, $\Phi$-Universal variable.

The momentum source term should be added into momentum equation while calculating porous domain. The source term consists of two parts: Viscous loss term and internal loss term. For simple uniform porous medium, the source term $S_{M, i}$ can be expressed as in equation (2):

$$
S_{M, i}=-\frac{\mu}{\alpha} u_{i}-\frac{K_{\text {loss }}}{2} \rho|\vec{u}| u_{i}
$$

Where, $\mu$-dynamic viscosity, $\alpha$-permeability coefficient, $\mathrm{K}_{\mathrm{loss}}$-resistance coefficient 。

The Darcy's law needs to be revised when $\mathrm{Re}_{\mathrm{p}}$ is greater than 10. In this paper, the Darcy-Brinkman Model ${ }^{[10]}$ is used as described in equation (3):

$$
-\frac{d_{p}}{d_{x}}=\frac{\mu}{K} u+\frac{c_{f}}{\sqrt{K}} \rho u^{2}
$$

Where, $\mathrm{K}$-permeability; $\mathrm{C}_{\mathrm{f}}$-inertia coefficient that:

According to the equations (2) and (3), it can be seen

$$
\alpha=K \quad K_{\text {loss }}=\frac{2 C_{f}}{\sqrt{K}}
$$

The process of heat transfer in porous medium follows the heat transfer formula, the effective thermal conductivity needs to be revised as follows:

$$
\lambda_{e}=\varphi \lambda_{f}+(1-\varphi) \lambda_{s}
$$

Where, $\varphi$-porosity, $\lambda_{\mathrm{f}}$ - fluid thermal conductivity, $\lambda_{\mathrm{s}}$-porous medium thermal conductivity, $\lambda_{\mathrm{e}}$-effective thermal conductivity.

The physical parameters of porous medium are listed in Table $1^{[11]}$. In order to evaluate the impact of the thermal conductivity of porous media on heat transfer, three types of porous medium $(\mathrm{Fe}, \mathrm{Al}$, and $\mathrm{Cu}$ ) were used in the simulations, with their thermal conductivity $\lambda_{s}$ of 20,220 and $400 \mathrm{~W} / \mathrm{m} / \mathrm{K}$, respectively.

Tab.1 Parameters of porous media

\begin{tabular}{|c|c|c|}
\hline $\boldsymbol{\varphi}$ & $\boldsymbol{K} / \mathbf{m} \mathbf{2}$ & $\boldsymbol{C}_{\boldsymbol{f}}$ \\
\hline 0.975 & $5.721 \times 10^{-7}$ & 0.015 \\
\hline 0.967 & $3.064 \times 10^{-7}$ & 0.016 \\
\hline 0.95 & $1.964 \times 10^{-7}$ & 0.017 \\
\hline
\end{tabular}


The physical parameters related to temperature (T) for molten salt in numerical simulation were described as follows:

$$
\begin{gathered}
\rho=-0.6365 T+2264 \\
C_{p}=0.1726 T+1395.6 \\
\lambda_{f}=0.0002 T+0.3896 \\
\eta=-1.47102747909196 \times 10-10 T 3+ \\
3.48238912393835 \times 10-7 T 2- \\
0.000277132299616017 T+ \\
0.0754000796626019
\end{gathered}
$$

Where, $\rho$-density, $\mathrm{kg} / \mathrm{m}^{3} ; C_{p}$-specific heat capacity, $\mathrm{J} /(\mathrm{kg} \cdot \mathrm{K}) ; \quad \lambda_{f}$-thermal conductivity; $\eta$-dynamic viscosity, $\mathrm{mPa} \cdot \mathrm{s} ; T$-temperature, $\mathrm{K}$.

\subsection{Boundary conditions}

In numerical simulation, the uniform velocity and temperature distribution were given at inlet cross $\operatorname{section}\left(v=V_{\mathrm{c}}, \quad T=T_{\mathrm{c}}=573.15 \mathrm{~K}\right)$, and the pressure boundary was defined as $p=p_{\text {out }}$ at the outlet cross section. The change of heat flux distribution in the outer wall of the receiver tube along the $\mathrm{x}$-axis was negligible, but highly dependent on the arrangement of the Linear Fresnel Reflector and the strength of solar radiation energy along the y-axis. To simulate the practical heat transfer, the heat flux distribution on the outer tube at $\mathrm{DNI}=1000 \mathrm{~W} / \mathrm{m} 2$ was applied in this paper, as shown in Fig. $2^{[12]}$ :

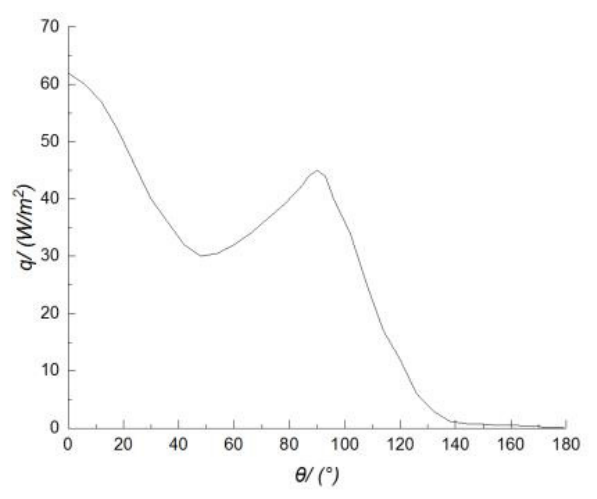

Fig.2. The circumferential Hheat flux distribution on the outer surface wall of receiver tube around the circumference

\subsection{Grid system and model validation}

Hexahedral structure was used for grid generation to fast convergence. Second-order upwind format was applied in the momentum equation, turbulent flow energy, the dissipative equation and energy equation, SIMPLE algorithm was applied to solve the equations and the residuals less than $10^{-6}$ were adopted as standard of convergence for the mass equation, momentum equation and energy equation. After numerical calculation, the velocity distribution and temperature distribution at the cross section were given and the average convective heat transfer coefficient could be calculated by $h=\frac{q}{T_{w, i}-T_{f, m}}$, where, $\mathrm{q}$-Average heat flux, $\mathrm{T}_{\mathrm{w}, \mathrm{i}}$-Average temperature of inner wall; $T_{f, m}$-average mass flow on the cross section; The heat transfer effect was evaluated by $\mathrm{N}_{\mathrm{u}}=\frac{h \mathrm{D}_{\mathrm{i}}}{\lambda_{\mathrm{f}}}$, and the comprehensive performance index was evaluated by $\mathrm{PEC}=\frac{\mathrm{N}_{\mathrm{u}} / \mathrm{N}_{\mathrm{u}}}{\left(\mathrm{f} / \mathrm{f}_{0}\right)^{1 / 3}}$ for the whole receiver tube. where $f$ is the average resistance coefficient,and the subscript number 0 stands for the number of tubes.

Grid independence test was carried out by increasing the number of grids to $80,000,100,000,150,000$ and 200,000 . The results became stable when the number of grids reached 150,000 . Based on this grid number and uniform heat flux on the outer wall of the clear receiver tube, the calculation results from the turbulent flow in the clear receiver tube was compared to the references, which revealed that the value of $\mathrm{Nu}$ was closer to Gnielinski correlation, with a maximum error 5\%. The maximum error of calculated $\mathrm{f}$ was less than $10 \%$ compared to the Filonenko correlation. These results indicated that the numerical simulation was effective to analyze the heat transfer characteristics in the molten salt-type receiver tube by using this modal and grid system herein.. In this paper, the porous medium with e $=0.975$ were applied to analyze the heat transfer based on the model and boundary conditions described in the literature[11], and the maximum errors for $\mathrm{Nu}$ and $\mathrm{f}$ were $5 \%$ and $15 \%$, respectively.

\section{Results and discussion}

\subsection{Flow field and temperature field characteristics analysis}

As shown in Fig.3, the temperature distributions at the cross section were given for two types under the conditions: Entering velocity $0.5 \mathrm{~m} / \mathrm{s}$, porous medium $\mathrm{Cu}$ with porosity e $=0.975$ was applied. From Fig.3.a-3.e, it can be seen that the temperature gradient was remarkable at the wall near surface when the filling rate $\left(\mathrm{Y}_{\mathrm{c}}\right)$ was very small for center filling situation. However, with the filling rate increasing, the temperature distribution at the cross section became uniform, and reached best performance when $Y_{c}=0.6$. While $Y_{c} \geq 0.8$, the temperature layers appeared, due to the enlargement of the total porous area with increasing filling rate which resulted in more homogeneous fluid distribution inside the porous area. When the fluid is heated by external non-uniformed heat flux, Temperature stratification tends to be formed. As shown in Fig. 3.f-3.j, the similar temperature layers were formed at $Y_{0} \geq 0.6$ and the uniform temperature distribution was observed at $Y_{o}=0.4$ for annular filling situation. When the tube was completely filled (Fig. 3n), the temperature distribution showed ring shaped layers at the entire cross 
section, with high temperature outside and low temperature inside.
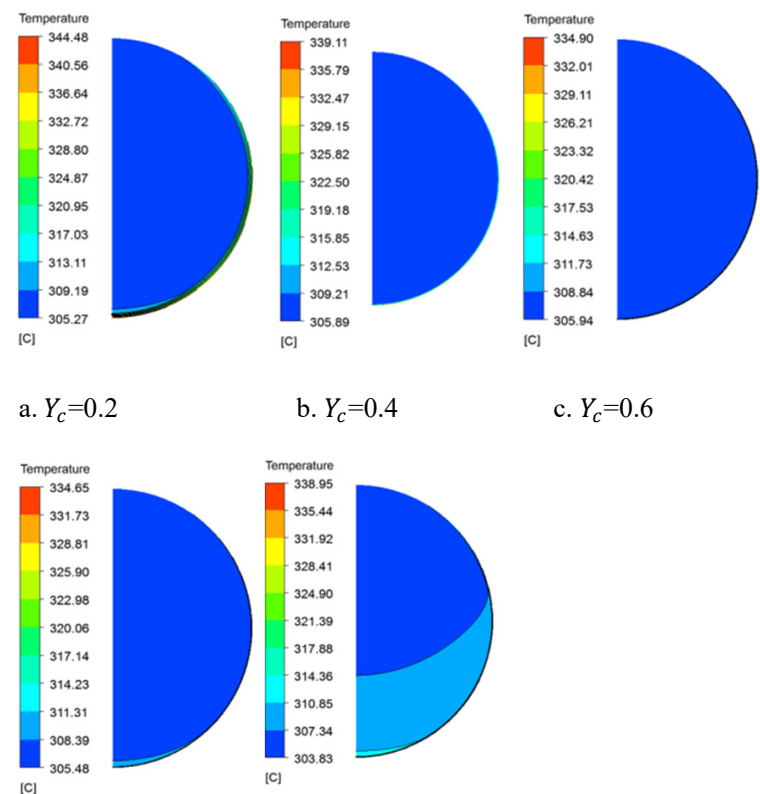

c. $Y_{c}=0.6$

$$
\begin{array}{ll}
\text { d. } Y_{c}=0.8 & \text { e. } Y_{c}=0.9
\end{array}
$$
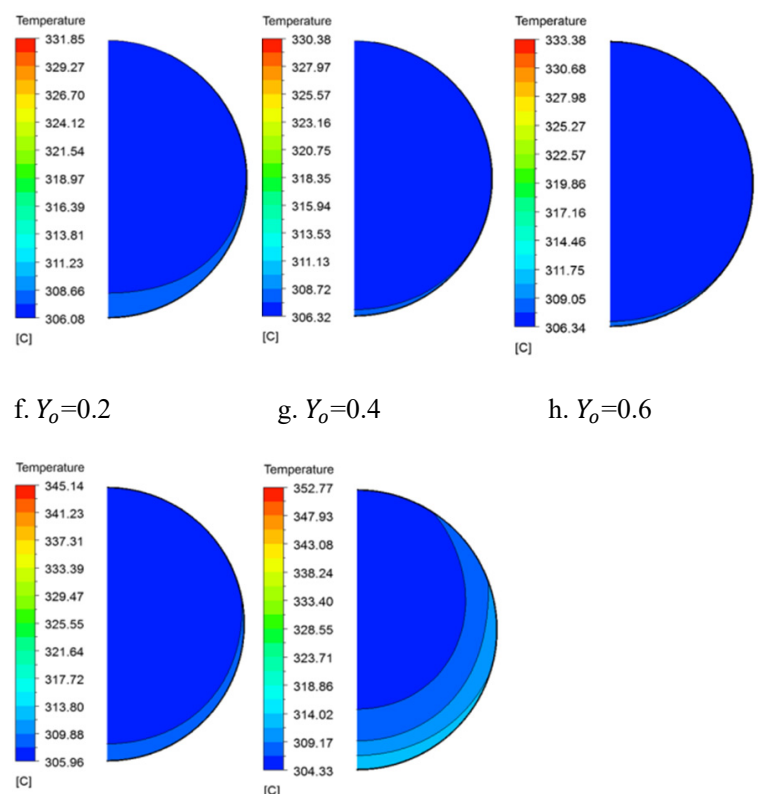

g. $Y_{o}=0.4$

h. $Y_{o}=0.6$

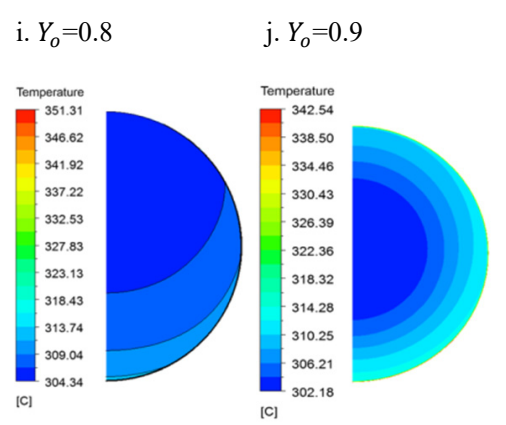

m. clear receiving tube

n. complete filling

Fig.3.Temperature distribution at cross section for different kinds filling

\subsection{Heat transfer characteristics and comprehensive performance analysis}

As shown in Fig. 4, Nu and PEC were varied with the change of filling rate, under the conditions: Entering velocity $0.5 \mathrm{~m} / \mathrm{s}, \mathrm{Cu}$ porous medium with porosity e $=0.975$. With the increase of filling rate, $\mathrm{Nu}$ increased firstly and then decreased for both filling types. For center filling, when $\mathrm{Y}_{\mathrm{c}}$ increased from 0 to $0.9, \mathrm{Nu}$ was consistently higher than the clear receiving tube $\left(\mathrm{Nu}_{0}\right)$. $\mathrm{Nu}$ could reach 118 and the heat transfer efficiency was 1.7 times that of the clear receiving tubes when $\mathrm{Y}_{\mathrm{c}}=0.8$. For annular filling, $\mathrm{Nu}$ was higher than the clear receiving tube $\left(\mathrm{Nu}_{0}\right)$ when $\mathrm{Y}_{\mathrm{o}}$ increased from 0.2 to 0.6 . $\mathrm{Nu}$ would reach 102 and the heat transfer efficiency was 1.5 times that of the clear receiving tubes when $Y_{o}=0.2$. For center filling, PEC was higher than 1 when $Y_{c}$ increased from 0.2 to 0.8 , and reached 1.34 when $Y_{c}=0.4$. However, PEC decreased with the increase of filling rate for annular filling, and reached 1.46 when $Y_{o}=0.1$.

According to Fig. 3, the variation of $\mathrm{Nu}$ with filling rate was closely related to the temperature distribution. High temperature field uniformity was benefit for obtaining better $\mathrm{Nu}$ performance, and vice versa. PEC increased with the filling rate increasing when the filling rate $\mathrm{Y}_{\mathrm{c}} \leq 0.4$ for center filling. When heat transfer liquid flowed in the tube, the liquid was squeezed by the porous media and flowed into the annular gap consequently, which result in the increase of velocity gradient near the wall. However, the improvement of heat transfer effect was greater than the increase of flow resistance. As a result, PCE would increase in this filling rate range. When filling rate increased further, PEC began to decrease because the decrease of annular gap led to a sharp increase of flow resistance. PEC was greater than 1 when the filling rate of annular filling $\mathrm{Y}_{\mathrm{o}} \leq 0.2$. When the filling rate increased further, more fluid would flow into the center which caused the increase of flow rate in the center. Then comprehensive performance would be reduced because the increase of center flow rate had no effect to enhance the heat transfer. It can be seen that there were optimal filling rates for both filling types to obtain the best heat exchange effect.

As shown in Fig. 5, and PEC both decreased when the porosity of filling medium increased. Therefore, the medium with lower porosity was good for enhancing heat transfer in practical applications. Fig. 6 showed how $\mathrm{Nu}$ changed with $\lambda_{\mathrm{s}}$ increasing $\left(\lambda_{\mathrm{s}}=\lambda_{\mathrm{f}} 、 20 、 220 、 400\right.$ $\mathrm{W} / \mathrm{m} / \mathrm{K}$ ) when porosity $\mathrm{e}=0.975, \mathrm{Y}_{\mathrm{c}}=0.8$ and $\mathrm{Y}_{\mathrm{o}}=0.2$. The results indicated that $\mathrm{Nu}$ was irrelevant to the thermal conductivity of the filling medium. This demonstrated that the impacts of porous medium on heat transfer enhancement were mainly due to the velocity field rebuilding. 


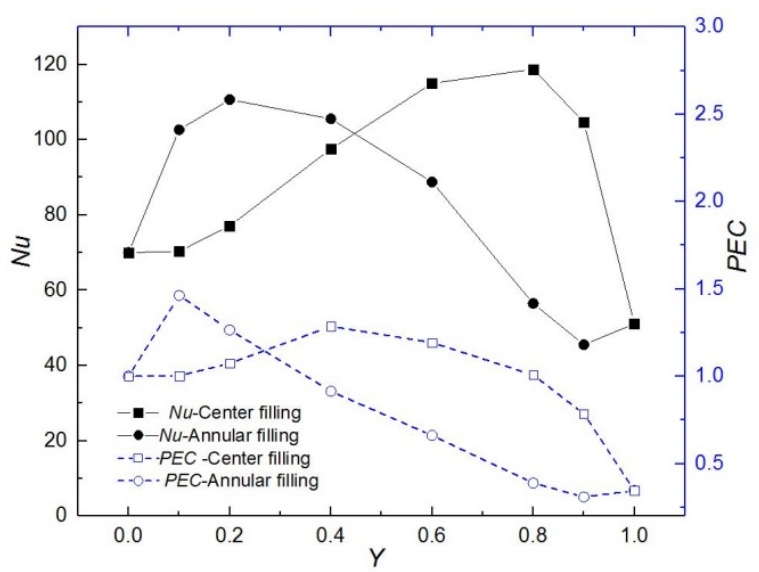

Fig.4. $N u$ and $P E C$ as functions of $Y$ for different kinds filling

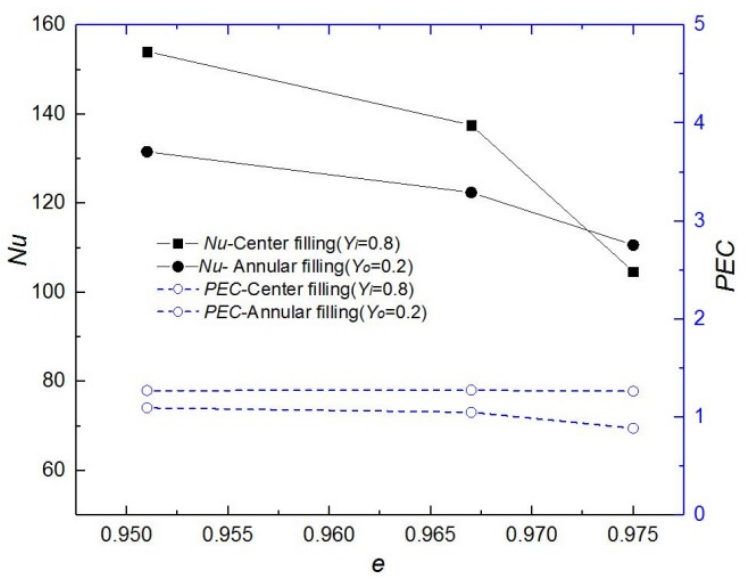

Fig.5. $N u$ and $P E C$ as functions of $\operatorname{Porosity}(e)$

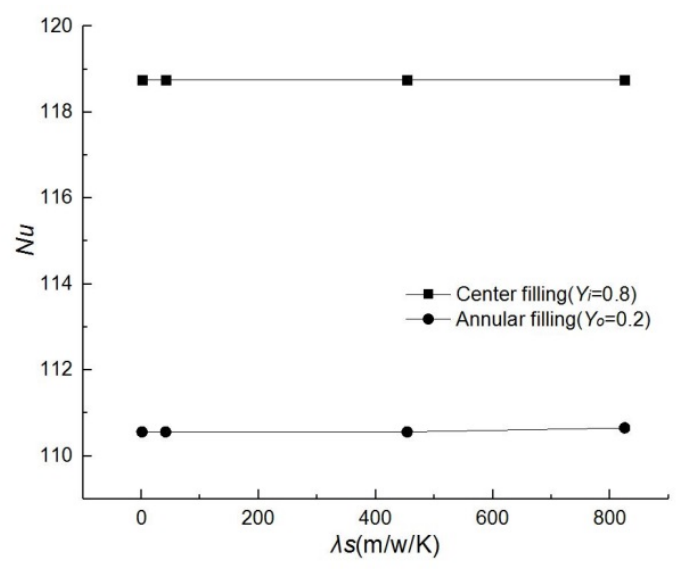

Fig.6.The relationship of $N u$ and $\lambda_{s}$ for different kinds filling

\subsection{Circumferential wall temperature analysis}

Fig.7.showed the correlation between maximal circumferential wall temperature difference (MCWTD) and filling rate at entering velocity of $0.5 \mathrm{~m} / \mathrm{s}$ with medium porosity e $=0.975$. The MCWTD decreased firstly and then increased with the increase of filling rate for both filling situations. Lowest MCWTD was $34.02^{\circ} \mathrm{C}$ at $Y_{C}=0.8$ for center filling and $27.92^{\circ} \mathrm{C}$ when $Y_{o}=0.4$ for annular filling, with a reduction of $15.88^{\circ} \mathrm{C}$ and $22^{\circ} \mathrm{C}$ compared to clear receiving tube, respectively. In general, the center filling had better performance than annular filling. According to Fig. 4, it can be seen that the circumferential wall temperature difference of the receiving tube was correlated to the temperature distribution uniformity of the fluids. The more uniform the cross sectional temperature distribution was, the corresponding circumferential wall temperature difference was smaller. When $\mathrm{Y}_{\mathrm{c}}=0.8$ for center filling and $Y_{o}=0.4$ for annular filling, the uniformity for the liquid temperature distribution was best and the circumferential temperature difference at cross section was lowest. In addition, the lowest circumferential wall temperature difference $\left(10.14^{\circ} \mathrm{C}\right)$ was observed for complete filling. However, it was not suitable for practical application because of high flow resistance. Consequently, it was better to adopt annular fillings with $\mathrm{Y}_{\mathrm{o}} \leq 0.4$ in practical applications for lower circumferential wall temperature difference.

Fig.8.showed the relation between the maximal circumferential wall temperature difference and $\lambda_{s}$ at entering velocity of $0.5 \mathrm{~m} / \mathrm{s}$ with porosity $\mathrm{e}=0.975$ and center filling $\mathrm{Y}_{\mathrm{c}}=0.8$ and annular filling $\mathrm{Y}_{\mathrm{o}}=0.2$. The thermal conductivity of the filling medium had little impact to maximal circumferential wall temperature difference, which further indicated that the velocity field rebuilding was the dominant factor in lowering the circumferential wall temperature difference in porous media. For annular filling, circumferential wall temperature difference was slightly decreased only when $\mathrm{Cu}$ was used for porous medium.

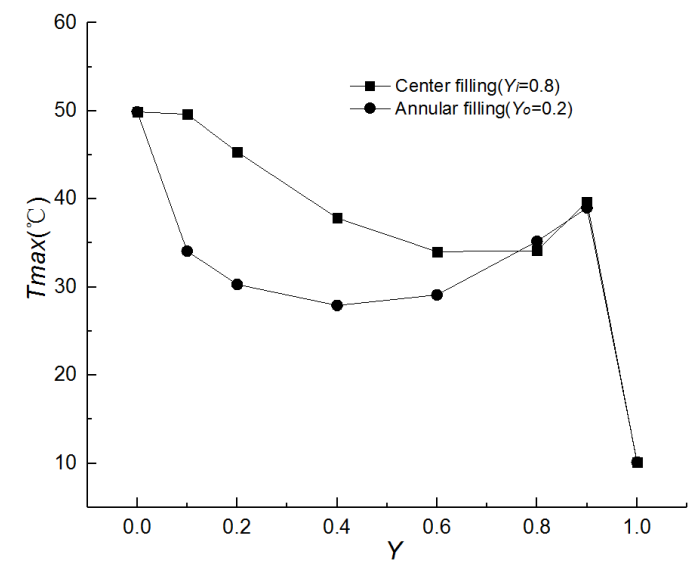

Fig.7.The relationship of the maximum circumferential wall temperature difference of the tube and $\mathrm{Y}$ for different filling types 


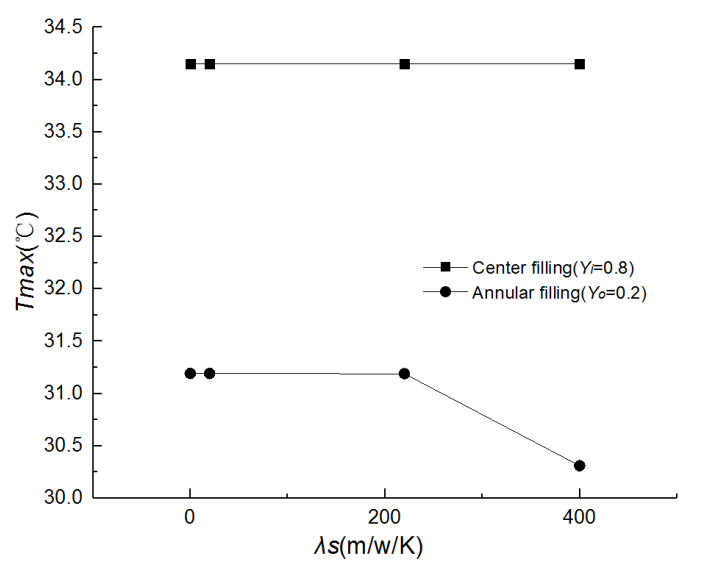

Fig.8. The relationship of the circumferential wall temperature difference and $\lambda_{s}$ for different filling types

\section{Conclusions}

Based on numerical simulation, this study analyzed the characteristics of heat transfer enhancement and wall temperature in the solar receiver tube by inserting porous media with different conditions (filling rate, porosity, thermal conductivity, etc.) in Linear Fresnel receiver tube using molten salt as HTF. Following conclusions were obtained in this study: (1)Nu increased firstly and then decreased when filling rate was increasing for both center filling and annular filling, and the optimal thermal performance were obtained when filling rate was 0.8 for center filling and 0.2 for annular filling. (2) The circumferential temperature difference decreased firstly and increased when filling rate was increasing for both center types of filling and the lowest circumferential temperature difference were obtained at filling rate of 0.8 for center filling and 0.4 for annular filling. (3) Both $\mathrm{Nu}$ and PEC decreased with the increase of porosity, but the thermal conductivity of porous medium had slight impact to $\mathrm{Nu}$ and circumferential temperature difference. According to systematic study of two filling types in impacting heat flow and transfer characteristics, it could give better understanding of the correlation between porous medium and heat transfer enhancement. Based on this study, development of reasonable filling methods for different heat transfer media, pipe wall materials and heat flow distribution outside the pipe is the future research direction for solar heat collecting tubes.

\section{References}

1. Haiwei Xu, Chun Chang, Qiang Yu, Study and Applications of the Melted Salt Technologies in Concentrating Solar Power Generation Systems[J]. Journal of Engineering for Thermal Energy and Power,2015, 30(05):659-665.

2. Qiu Yu, Yaling He, Zedong Cheng, et al. Study on optical and thermal performance of a linear Fresnel solar reflector using molten salt as HTF with MCRT and FVM methods[J]. Applied Energy, 2015,146.
3. Chun Chang, Shidong LI, Xin Li, et al. Enhanced Heat Transfer Characteristics of Molten Salt in Solar Thermal Absorber Tubes With Circumferentially Non-uniform Heat Flux Boundary Condition [ $\mathrm{J}]$.Proceedings of the CSEE, 2014, 34(20):3341-3346.

4. Weishu Wang; Lin Fu; Zhen Li, et al. Investigation on heat transfer of molten salt in bellows with twist tape inserts[J]. Cryogenics \& Superconductivity, 2017, 45(10):58-64.

5. Weiwei YAN, Shifu GE, Yang LI. Numerical simulation on heat transfer enhancement in parabolic trough solar collector of DSG systems[J]. Journal of Chinese Society of Power Engineering, 2013, 33(07):550-554.

6. Pei Wang, Deyou Liu, Chang Xu, et al. Numerical Research on Enhanced Heat Transfer by Inserting Metal Foams in the Receiver Tube of the DSG System[J] Proceedings of the CSEE, 2012,32(23):83-89.

7. Pei Wang, Deyou Liu, Chang $\mathrm{Xu}$, et al.Three dimensional numerical simulation of heat transfer enhancement using porous media in solar vacuumed collector[J] Energiae Solaris Sinica, 2012,33(12):2105-2110.

8. K. Ravi Kumar, K.S. Reddy, Thermal analysis of solar parabolic trough with porous disc receiver[J]. Applied Energy,2008, 86(9).

9. Zhangjing Zheng, Yang Xu, Yaling He. Thermal analysis of a solar parabolic trough receiver tube with porous insert optimized by coupling genetic algorithm and CFD $[\mathrm{J}]$.Science China (Technological Sciences),2016,59(10):1475-1485.

10. Yuan Tian, Changying Zhao, Zengyao Li, et al.Flow and heat transfer in metal foam filled popes under two extended darcy models[J]. Journal of Engineering Thermophysics, 2008(08):13801382 .

11. Zhifeng Huang. Experimental and numerical studies of enhancing heat transfer in a tube by inserting porous media at the core[D].Huazhong University of Science and Technology,2010.

12. Yu Qiu; Yaling He. Optical performance investigation and optimization of a linear Fresnel reflector solar collector[J]. Journal of Engineering Thermophysics, 2015,36(12):2551-2556. 